\title{
Attacks of immobility caused by diet or exercise? The mystery of periodic paralyses
}

\author{
Jacob Levitt, MD; Patrick Cochran, PhD, CSD; and Janet Jankowiak, MD
}

In dreams, people often experience the terror of being unable to move, as if frozen. In traumatic situations, some people become "paralyzed with fear," unable to flee. For people with periodic paralysis, this temporary freezing occurs in real life, often brought on by exercise, cold, or what they ate. This rare disorder is due to a problem at chemical sites (known as ion channels) that disrupt normal muscle function. Periodic paralysis includes several different diseases (see below, About Periodic Paralyses) that share some common features: (1) certain events trigger weakness or stiffness, (2) muscle function is often normal between attacks, and (3) the underlying problem may be due to a genetic change (mutation) at the ion channel in the muscle.

Although periodic paralysis is rare, other common disorders of the nervous system also feature intermittent attacks, such as migraine headaches and epilepsy. Understanding the correlation between the clinical signs of periodic paralysis and its underlying genetics (how it is inherited) may help researchers find targeted drug treatments for it and the more common disorders.

In this issue of Neurology, Miller at al. (2004;63:1647-1655) present the largest series of patients with periodic paralysis, collected over a 15year period: 71 patients with hypokalemic periodic paralysis, 99 with hyperkalemic periodic paralysis, and 56 with paramyotonia congenita. The specific signs of each type of periodic paralysis (phenotype) were compared with the underlying genetic composition (genotype). The authors hoped that finding correlations between phenotype and genotype might eventually help doctors predict disease symptoms and severity based on a blood test of the gene.

From this study, Miller et al. highlight the distinguishing clinical features of the periodic paralyses (table). As their names suggest, the blood level of potassium during an attack of weakness or stiffness is the key feature.

In general, the periodic paralyses strike early in life. The most common of these is hypokalemic periodic paralysis, in which attacks are triggered by "sweets" and salt as well as by exercise. Patients note that the disease is worst during the teenage years. This might be due to hormonal changes, an unpredictable teenage diet, or inexperience with the disease. In contrast, attacks of hyperkalemic periodic paralysis tend to be shorter, but more frequent. In paramyotonia congenita, if weakness occurs, it happens after a bout of myotonia (when muscles are stiff and won't relax).

Miller et al. were able to identify specific gene mutations in two-thirds of the cases. In the third of cases where a mutation was not identified, the clinical presentation was less typical. These patients tended to be older when the attacks began $(>20$ years old), and diet did not consistently trigger attacks, although exercise was a common trigger. They also seemed to be spared permanent muscle weakness. The authors propose that periodic paralysis patients without known mutations may have mutations in yet other ion channels or may be due to other metabolic disorders.

A major concern of younger patients with periodic paralysis is the threat of permanent muscle weakness of the hips, thighs, and shoulders.

Miller et al., using genetic testing, were unable to predict with certainty who will or will not escape permanent muscle weakness. However, given today's rapid advances in genetic technology, their important work lays the foundation for future therapies, and perhaps, even a cure.

Table Clinical features of the familial periodic paralyses (PP)

\begin{tabular}{|c|c|c|c|}
\hline Feature & Hypokalemic PP & Hyperkalemic PP & $\begin{array}{l}\text { Paramyotonia } \\
\text { congenita }\end{array}$ \\
\hline Age at onset & 5 to $20 \mathrm{yr}$ & $<10 \mathrm{yr}$ & Infancy \\
\hline Attack duration & Hours to over a day & Minutes to hours & Minutes to hours \\
\hline \multirow[t]{3}{*}{ Triggers } & Exercise & Exercise & Exercise \\
\hline & "Sweets" (high carb) & Hunger & Cold \\
\hline & Salt & Potassium-rich food & \\
\hline Myotonia & No & Sometimes & Always \\
\hline $\begin{array}{l}\text { Permanent } \\
\text { weakness }\end{array}$ & Yes (not always) & Yes (not always) & Yes (not always) \\
\hline Potassium level & Low & Normal to high & Normal \\
\hline $\begin{array}{l}\text { Response to } \\
\text { potassium }\end{array}$ & Relieves acute weakness & Causes weakness & Variable \\
\hline
\end{tabular}


The patient's point of view

"Overcome by a sense of profound weakness, a feeling of helplessness, unable to move, yet conscious of activities around you-awake, but trapped in an unmovable body." These are typical descriptions given by individuals suffering from periodic paralysis. In the early stages, children may discover they can no longer run or keep up with their classmates. Severe muscle stiffness, unpredictable bouts of paralysis or the onset of permanent weakness limit the individual's ability to perform normal daily tasks, jeopardizing employment and personal relationships, and the overall quality of life.

\section{Description and onset of symptoms}

It is estimated that fewer than 1 in 100,000 individuals are affected worldwide. Periodic paralysis is classified as a "rare disease," meaning that fewer than 200,000 cases are known to exist in the United States. In the classic forms of periodic paralysis, the first episode typically occurs by age 20 years. Girls and boys are equally at risk. Significant variability exists between individuals, including length of attacks, which range from a few minutes to several days. Attacks can be unpredictable or result from specific activities, such as rest after exercise or exposure to cold. Certain foods can also provoke an attack. Permanent weakness, especially in the hips and thighs, can develop later in life in all forms of periodic paralysis.

\section{What is periodic paralysis?}

Periodic paralysis is a collection of disorders caused by changes (mutations) in the genetic recipe for the composition of ion channels in muscle. lon channels are openings that allow the transfer of ions through the cell membrane.

Ion channels control many of life's processes. Periodic paralysis is associated with mutations in calcium, sodium, or potassium channels, causing muscle dysfunction. There

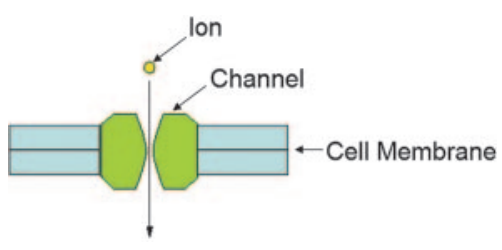

to cold. Because sodium channel mutations cause both PMC and hyperKPP, these two diseases are often found in the same patient.

\section{Andersen-Tawil syndrome (ATS)}

is a $50-50$ chance of a parent passing the mutation on to a child. However, cases where neither parent has the condition do occur.

\section{Hypokalemic periodic paralysis (hypoKPP)}

HypoKPP is the most common form of periodic paralysis, mainly caused by mutations in the calcium or sodium channels. Symptoms include profound weakness and a decrease (hypo $=$ low) in blood potassium $(\mathrm{K})$ during attacks. Patients remain fully conscious during attacks, but may be totally paralyzed in their arms and legs. Sometimes muscles needed for breathing may be affected. Lifethreatening irregularities in heartbeat may occur due to low potassium. Sometimes, an overactive thyroid gland can cause symptoms of hypoKPP (thyrotoxic periodic paralysis, or TPP). In these cases, correction of the thyroid problem cures the periodic paralysis. TPP is not inherited.

\section{Hyperkalemic periodic paralysis (hyperKPP)}

HyperKPP is a problem of the muscle sodium channel. Weakness usually lasts under an hour but can persist for over a day. Common triggers include exercise, fasting, or too much potassium-rich foods (e.g., bananas, orange juice). Myotonia (muscle stiffness) is also a feature. Some patients may experience only a few attacks of weakness in their lifetime, while others have reported daily attacks of generalized weakness. Potassium is generally high during attacks, and problems with heart rhythm can result.

\section{Paramyotonia congenita (PMC)}

The defining symptoms in PMC are muscle stiffness that increases with continued activity (paramyotonia) and severe myotonia when exposed
While not reported by Miller et al., ATS is a type of periodic paralysis caused by mutations in the potassium channel, which is found in skeletal and heart muscle. ATS is defined by periodic paralysis, irregular heartbeat, and abnormal physical features. Because dangerous heart rhythms occur with ATS, any patient with periodic paralysis should have his heart rhythm checked with an EKG.

\section{Management and treatment of periodic paralysis}

Periodic paralysis is commonly managed with a healthy combination of medication and the identification and avoidance of attack triggers. An ounce of prevention is worth a pound of cure! For hypoKPP, patients should avoid foods like pizza, pasta, and candy. HyperKPP patients must beware of foods high in potassium. PMC patients should avoid the cold. There is significant variability in sensitivity to dietary components. Acute attacks of weakness can be relieved by potassium for hypoKPP patients or by eating sugary foods for hyperKPP. Acetazolamide or dichlorphenamide helps prevent attack frequency and severity but will not make an acute attack of weakness or stiffness go away. More tips on managing periodic paralysis can be found at www.periodicparalysis.org (click on PPA Doctor), but only use this information under the advisement of your treating physician.

\section{For more information}

Periodic Paralysis Association www.periodicparalysis.org/ Gene Reviews www.geneclinics.org Consortium for Clinical Investigation of Neurologic Channelopathies www. rarediseasesnetwork. org/cinch/index.htm 


\section{Neurology}

Attacks of immobility caused by diet or exercise? The mystery of periodic paralyses

Jacob Levitt, Patrick Cochran and Janet Jankowiak

Neurology 2004;63;E17-E18

DOI 10.1212/01.WNL.0000146275.91221.65

\section{This information is current as of November 8,2004}

\section{Updated Information \&} Services

Permissions \& Licensing

Reprints including high resolution figures, can be found at: http://n.neurology.org/content/63/9/E17.full

Information about reproducing this article in parts (figures,tables) or in its entirety can be found online at:

http://www.neurology.org/about/about_the_journal\#permissions

Information about ordering reprints can be found online:

http://n.neurology.org/subscribers/advertise

Neurology ${ }^{\circledR}$ is the official journal of the American Academy of Neurology. Published continuously since 1951, it is now a weekly with 48 issues per year. Copyright . All rights reserved. Print ISSN: 0028-3878. Online ISSN: 1526-632X.

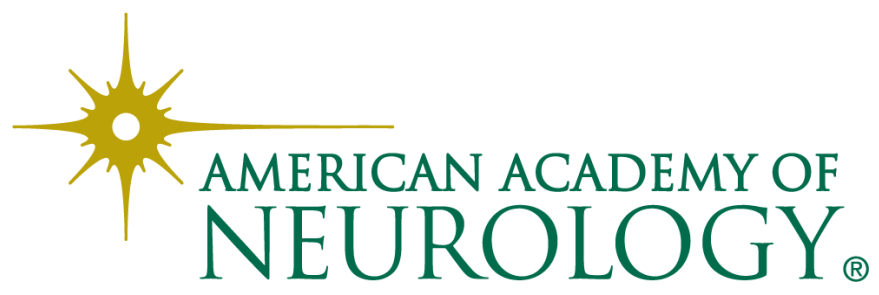

